\title{
Single-Electron and Single-Photon Sensitivity with a Silicon Skipper CCD
}

\author{
Javier Tiffenberg, ${ }^{1, *}$ Miguel Sofo-Haro, ${ }^{2,1}$ Alex Drlica-Wagner, ${ }^{1}$ Rouven Essig, ${ }^{3}$ Yann Guardincerri, ${ }^{1, \dagger}$ \\ Steve Holland, ${ }^{4}$ Tomer Volansky, ${ }^{5}$ and Tien-Tien $\mathrm{Yu}^{6}$ \\ ${ }^{1}$ Fermi National Accelerator Laboratory, P.O. Box 500, Batavia, Illinois 60510, USA \\ ${ }^{2}$ Centro Atómico Bariloche, CNEA/CONICET/IB, Bariloche R8402AGP, Argentina \\ ${ }^{3}$ C.N. Yang Institute for Theoretical Physics, Stony Brook University, Stony Brook, New York 11794, USA \\ ${ }^{4}$ Lawrence Berkeley National Laboratory, One Cyclotron Road, Berkeley, California 94720, USA \\ ${ }^{5}$ Raymond and Beverly Sackler School of Physics and Astronomy, Tel-Aviv University, Tel-Aviv 69978, Israel \\ ${ }^{6}$ Theoretical Physics Department, CERN, CH-1211 Geneva 23, Switzerland
}

(Received 4 June 2017; published 26 September 2017)

\begin{abstract}
We have developed ultralow-noise electronics in combination with repetitive, nondestructive readout of a thick, fully depleted charge-coupled device (CCD) to achieve an unprecedented noise level of $0.068 e^{-} \mathrm{rms} /$ pixel. This is the first time that discrete subelectron readout noise has been achieved reproducible over millions of pixels on a stable, large-area detector. This enables the contemporaneous, discrete, and quantized measurement of charge in pixels, irrespective of whether they contain zero electrons or thousands of electrons. Thus, the resulting CCD detector is an ultra-sensitive calorimeter. It is also capable of counting single photons in the optical and near-infrared regime. Implementing this innovative non-destructive readout system has a negligible impact on CCD design and fabrication, and there are nearly immediate scientific applications. As a particle detector, this CCD will have unprecedented sensitivity to low-mass dark matter particles and coherent neutrino-nucleus scattering, while future astronomical applications may include direct imaging and spectroscopy of exoplanets.
\end{abstract}

DOI: 10.1103/PhysRevLett.119.131802

Introduction.-Charge-coupled devices (CCDs) are essential for photon and other particle detection in many industrial and scientific applications, e.g., [1-4]. CCD sensors rely on the photoelectric effect to absorb incident photons in a silicon substrate and generate electron-hole pairs [2]. Energetic photons $(E \gtrsim 10 \mathrm{eV})$ produce multiple electron-hole pairs that allow for energy measurement, while lower energy photons may generate one or a few electron-hole pairs. Massive particles can create electronhole pairs either by directly interacting with valence-band electrons or by scattering off of silicon nuclei. In each case, precision measurements are limited by the readout noise of the CCD electronics. Readout noise is added to the video signal by the CCD output amplifier [4]. While correlated double sampling [5] dramatically reduces highfrequency readout noise, low-frequency readout noise has remained a fundamental limitation for precision singlephoton-electron counting in CCDs.

In conventional scientific CCDs, low-frequency readout noise results in rms variations in the measured charge per pixel of $\sim 2 e^{-} \mathrm{rms} /$ pixel [6,7]. Janesick et al. [8] proposed that low-frequency readout noise could be reduced by using a floating gate output stage [9] to perform repeated

Published by the American Physical Society under the terms of the Creative Commons Attribution 4.0 International license. Further distribution of this work must maintain attribution to the author(s) and the published article's title, journal citation, and DOI. measurements of the charge in each pixel. This multiple readout technique was implemented as a "Skipper CCD" $[8,10]$; however, the performance of these early detectors was limited by charge generation effects [11]. In this Letter, we utilized improvements in the isolation of external noise sources as well as slight modifications to the Skipper CCD of [11] to achieve a drastic reduction in low-frequency readout noise to the level of $0.068 e^{-} \mathrm{rms} /$ pixel after 4000 samples per pixel (Fig. 1). At this noise level, the probability that the charge per pixel is misestimated by $>0.5 e^{-}$is $p \sim 10^{-13}$. This represents the first accurate single-electron counting on a large-format $(4126 \times 866$ pixel $)$ silicon detector. (In contrast, $0.18 e^{-} \mathrm{rms} /$ pixel has been demonstrated for a $4 \times 4$ pixel miniarray of silicon depleted field effect transistors [12].)

Ultralow readout noise and stable linear gain allows the Skipper CCD to measure charge at the accuracy of individual electrons simultaneously in pixels with single electrons and with thousands of electrons. This makes the Skipper CCD the most sensitive and robust electromagnetic calorimeter that can operate above liquid nitrogen temperatures. The Skipper CCD can also count individual optical and near-infrared photons. Because nondestructive readout is achieved without any major modification to the CCD fabrication process, this new technology can be directly implemented in existing CCD manufacturing facilities.

Technical Description.-CCD detector: The detector studied here is a $p$-channel CCD fabricated on high resistivity ( $\sim 10 \mathrm{k} \Omega \mathrm{cm}), n$-type silicon that is fully depleted at a substrate voltage of $40 \mathrm{~V}$. The sensor is $200 \mu \mathrm{m}$ thick 


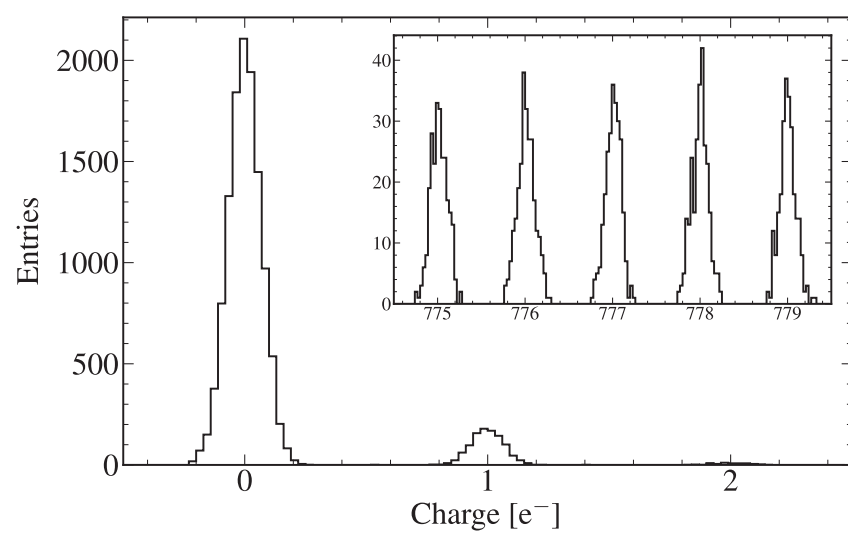

FIG. 1. Single-electron charge resolution using a Skipper CCD with 4000 samples per pixel (bin width of $0.03 e^{-}$). The measured charge per pixel is shown for low (main) and high (inset) illumination levels. Integer electron peaks can be distinctly resolved in both regimes contemporaneously. The $0 e^{-}$peak has rms noise of $0.068 e^{-} \mathrm{rms} /$ pixel while the $777 e^{-}$peak has $0.086 e^{-} \mathrm{rms} /$ pixel, demonstrating single-electron sensitivity over a large dynamical range. The Gaussian fits have $\chi^{2}=22.6 / 22$ and $\chi^{2}=19.5 / 21$, respectively.

and composed of $15 \times 15 \mu \mathrm{m}$ square pixels arranged in a $4126 \times 866$ array (Table I). The sensor is operated at $140 \mathrm{~K}$ to reduce the number of electrons promoted to the conduction band by thermal fluctuations ("dark current") $[13,14]$. (The operating temperature could be lowered to $\sim 100 \mathrm{~K}$ before charge-transfer efficiency is significantly reduced.)

Figure 2 shows a schematic of the Skipper CCD output stage [11]. At $t_{0}$, all charge is drained from the sense node (SN) to $V_{\text {drain }}$ by applying a pulse to the dump gate (DG), and the SN voltage is restored to $V_{\text {ref }}$ with a pulse to the reset gate (RG). At $t_{1}$, raising the summing-well gate (SG) phase transfers the charge packet to the $\mathrm{SN}$, concluding the readout of the first sample. To take the second sample, the output gate $(\mathrm{OG})$ and SG phase are lowered at $t_{2}$, moving the charge packet in the SN back under the SG phase. A pulse to the RG restores the $\mathrm{SN}$ reference voltage. This cycle is repeated to remeasure the same charge packet.

TABLE I. Skipper CCD detector characteristics.

\begin{tabular}{lcc}
\hline \hline Characteristic & Value & Unit \\
\hline Format & $4126 \times 866$ & pixels \\
Pixel scale & 15 & $\mu \mathrm{m}$ \\
Thickness & 200 & $\mu \mathrm{m}$ \\
Operating temperature & 140 & Kelvin \\
Number of amplifiers & 4 & \\
Dark current ${ }^{\mathrm{a}}$ & $<10^{-3}$ & $e^{-} / \mathrm{pixel} / \mathrm{day}$ \\
Readout time (1 sample) & 10 & $\mu \mathrm{s} / \mathrm{pixel} / \mathrm{amp}$ \\
Readout noise (1 sample) & 3.55 & $e^{-\mathrm{rms} / \mathrm{pixel}}$ \\
Readout noise (4000 samples) & 0.068 & $e^{-} \mathrm{rms} / \mathrm{pixel}$ \\
\hline \hline
\end{tabular}

${ }^{\mathrm{a}}$ The upper limit on dark current comes from measurements on a similar CCD used by the DAMIC experiment [15].

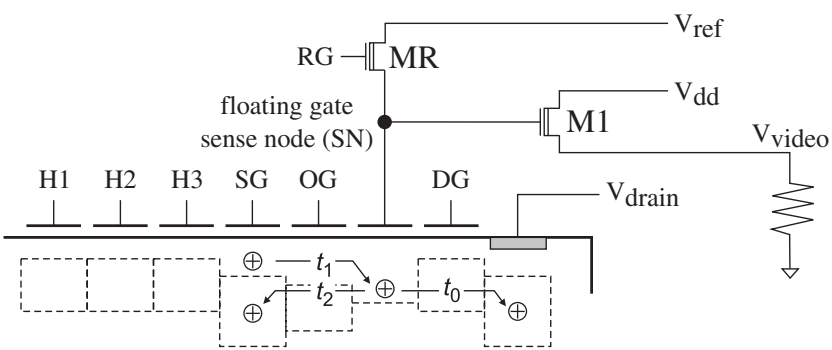

FIG. 2. Schematic of the Skipper CCD output stage. H1, H2, and $\mathrm{H} 3$ are the horizontal register clock phases. MR is a switch to reset the sense node to $V_{\text {ref. }}$ M1 is a MOSFET in a source follower configuration. Because of its floating gate, the Skipper CCD readout performs a nondestructive measurement of the charge at the SN.

The CCD is divided into four regions of $2063 \times 433$ pixels, and each region is read out by an amplifier with a distinct readout design. The most important difference between the four readout amplifiers is the size of the floating gate. Smaller floating gates have smaller capacitance and higher gain, but can be subject to charge transfer inefficiency and reduced full-well capacity. However, none of the designs tested showed adverse effects from reducing the size of the readout structures. The results discussed here are from the readout stage with the smallest $(15 \times 4 \mu \mathrm{m})$ floating gate. Additional improvements in gain are expected by further reducing the size of the floating gate, which will be explored in future generations of Skipper CCDs.

The output amplifiers have an impedance of $\sim 2 \mathrm{k} \Omega$ and can only drive signals for a few centimeters before significant degradation. To reduce the amplifier load, junction gate field-effect transistor (JFET) source followers that operate at low temperatures $(\sim 120 \mathrm{~K})$ were placed next to each analog output. The JFETs lower the output impedance, so signals can be driven over a $50 \mathrm{~cm}$ flex circuit and reach precision operational amplifiers at room temperature. These operational amplifiers have a noise density of $1.1 \mathrm{nV} / \sqrt{\mathrm{Hz}}$ and reduce the impact of internal noise sources in the readout electronics.

Readout electronics: The readout electronics are based on the Monsoon system developed for the Dark Energy Camera $[16,17]$. This system can be adapted to independently control the extra gates in the Skipper CCD and simultaneously digitize the four amplifier channels. To fully benefit from the multiple sampling capabilities of the Skipper CCD, the noise for each sample must be uncorrelated with the noise of the other samples. Although the electronics initially showed high levels of correlated noise, this was resolved by replacing all switching power supplies with low-noise laboratory power supplies with ripple noise $V_{\text {rms }}<350 \mu \mathrm{V}$ and peak-to-peak ripple voltage $V_{\mathrm{pp}}<2 \mathrm{mV}$ [18].

Signal processing and performance: One major advantage of the nondestructive readout technique is that individual samples are uncorrelated measurements of the 
charge in each pixel. For uncorrelated Gaussian readout noise, the standard deviation, $\sigma$, of the effective readout noise distribution after averaging $N$ samples per pixel is

$$
\sigma=\frac{\sigma_{1}}{\sqrt{N}}
$$

where $\sigma_{1}$ is the standard deviation of the readout noise for a single sample of the pixel. Figure 3 shows that the measured performance of the Skipper CCD closely follows this prediction, deviating only slightly for $N \gtrsim 2000$ samples. Thus, more sophisticated signal processing techniques are unnecessary.

Another advantage of nondestructive readout is that one can dynamically configure the number of samples taken per pixel. This allows the readout noise to be adjusted on a per pixel basis. The simplest application of this procedure is to repeatedly sample a predetermined subset of pixels where low readout noise is desired, while reading out the rest of the detector quickly with fewer samples per pixel. However, it is also possible to design sampling schemes that are based on the value of a pixel measured from the first readout. Thus, pixels containing more charge can be read fewer times. Dynamic sampling schemes can greatly reduce the readout time for the Skipper CCD when subelectron noise is not required in all pixels simultaneously.

Readout time: The Skipper CCD studied here has a single-sample readout noise of $\sigma_{1}=3.55 e^{-} \mathrm{rms} / \mathrm{pixel}$ with a readout time of $10 \mu \mathrm{s} / \mathrm{pixel} / \mathrm{sample}$. A readout noise of $\sigma<0.1 e^{-} \mathrm{rms} /$ pixel requires $\sim 1200$ samples per pixel, corresponding to a readout time of $12 \mathrm{~ms} /$ pixel. The wide-format detector described here can be read in three hours with four amplifiers. The readout time scales linearly with the number of samples and inversely with the square of the readout noise. Several modifications can further reduce the readout time. (1) The single-sample readout

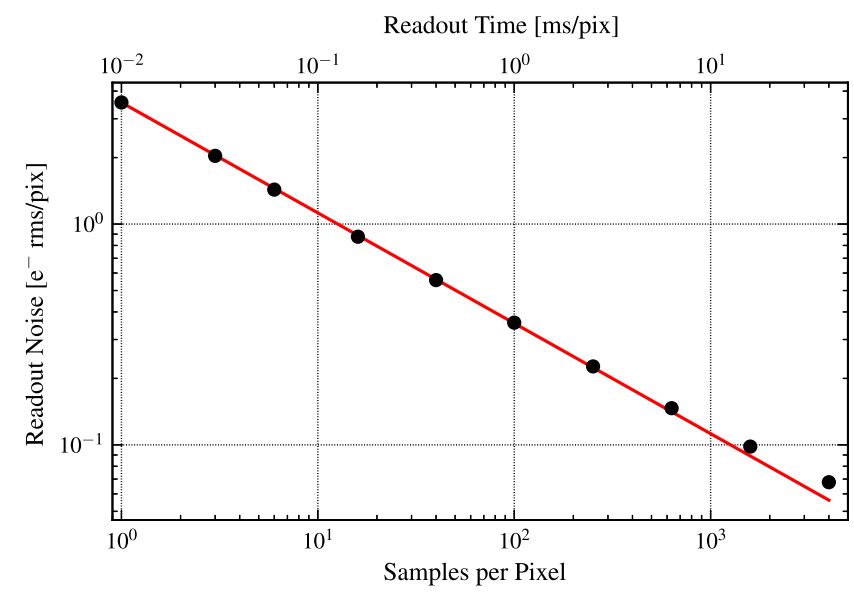

FIG. 3. Readout noise as a function of the number of nondestructive readout samples per pixel for the Skipper CCD. Black points show the rms of the empty-pixel distribution as a function of the number of averaged samples. The red line is the theoretical expectation assuming independent, uncorrelated samples [Eq. (1)]. noise and readout time can be decreased by using lower capacitance amplifiers with higher gain. Current CCDs using these amplifiers routinely reach $2 e^{-} \mathrm{rms} /$ pixel with a readout time of $4 \mu \mathrm{s} / \mathrm{pixel} / \mathrm{sample}$ [7]. Thus, one could reach $\sigma<0.1 e^{-} \mathrm{rms} /$ pixel 7.5 times faster than the device studied here. (2) The number of readout channels and amplifiers can be increased. Commercial devices are available with 16 amplifiers [19], and sensors with $>48$ on-chip amplifiers are well within the reach of existing technology [20]. The readout time decreases linearly with the number of amplifiers.

A combination of these two techniques can reduce the Skipper CCD readout time by a factor of $\mathcal{O}(100)$. In addition, a frame-shifting readout allows for readout of concurrent exposures. Finally, as previously discussed, dynamic sampling schemes can greatly reduce the readout time when only a fraction of the pixels require ultralow readout noise.

Impact of dark current: For detectors with subelectron readout noise, the dark current can become the dominant source of background in rare-event searches and limits the energy or charge threshold. A Skipper CCD with readout noise $\sigma \lesssim 0.1 e^{-} \mathrm{rms} /$ pixel will misclassify a pixel with $n$ electrons as having $n+1$ electrons with a probability of $p \sim 3 \times 10^{-7}(5 \sigma)$. This probability can be further reduced by selecting pixels with measured values that are within $3 \sigma$ from an integer charge (i.e., selecting pixels with measured values between $n-0.3$ and $n+0.3$, where $n$ is any nonnegative integer). By applying this quality cut, the misclassification probability is reduced to $p \sim 10^{-12}(\sim 7 \sigma)$, while the efficiency is kept above $99.7 \%$. Thus, readout noise no longer limits the energy or charge threshold.

The number of dark-current electrons that accumulate in a pixel is expected to follow a Poisson distribution with a mean equal to the dark-current rate multiplied by the exposure time. The minimum exposure time is set by the readout time. The Skipper CCD described here can be read out in three hours with a readout noise of $\sigma=$ $0.1 e^{-} \mathrm{rms} /$ pixel. Current experiments using fully depleted scientific CCDs similar to the Skipper CCD studied here have measured an upper limit on the dark-current rate of $\lesssim 10^{-3} e^{-} \mathrm{pixel}^{-1} / \mathrm{day}^{-1}$ [15], with no evidence for deviations from a Poisson distribution. Assuming the counts from the dark current follow a Poisson distribution, the upper limit on the expected number of pixels with an accumulated charge of $\geq 1 e^{-}\left(\geq 2 e^{-}\right)$is $\sim 450(\sim 0.03)$ per exposure. However, the theoretically expected dark-current rate for a CCD operating at $120 \mathrm{~K}$ is $\sim 10^{-7}$ [4], which would predict 0.04 pixels with $\geq 1 e^{-}$and $O\left(10^{-10}\right)$ pixels with $\geq 2 e^{-}$per exposure.

For dark-matter searches, the most important parameter is the charge or energy threshold, which should be set as low as possible to capture as many dark-matter events as possible [21] (the lowest possible threshold is set by the silicon band gap of $1.1 \mathrm{eV}$ ). Table II shows the expected dark-current contribution for a putative $100 \mathrm{~g}$ dark-matter detector consisting of 80 3.5-Mpix Skipper CCDs with a 
TABLE II. Number of pixels expected to exceed a given charge threshold as a function of the CCD dark current, assuming an experiment with a total exposure of $0.1 \mathrm{~kg}$ years. We assume that 80 Skipper CCDs each with $4126 \times 866$ pixels are read in 1.5 hours. A dark current of $10^{-3} e^{-}$pixel $^{-1} /$ day $^{-1}$ corresponds to the upper limit reported by DAMIC [15], while $10^{-5} e^{-}$pixel $^{-1} /$ day $^{-1}$ would allow a threshold of $2 e^{-}$in rare events searches, and $10^{-7} e^{-}$pixel $^{-1} / \mathrm{day}^{-1}$ is the theoretically expected rate.

\begin{tabular}{lccc}
\hline \hline $\begin{array}{l}\text { Dark current } \\
\left(e^{-} \text {pixel }^{-1} / \text { day }^{-1}\right)\end{array}$ & $\begin{array}{l}\geq 1 e^{-} \\
(\text {pixel) }\end{array}$ & $\begin{array}{l}\geq 2 e^{-} \\
(\text {pixel) }\end{array}$ & $\begin{array}{l}\geq 3 e^{-} \\
(\text {pixel })\end{array}$ \\
\hline $10^{-3}$ & $1 \times 10^{8}$ & $3 \times 10^{3}$ & $7 \times 10^{-2}$ \\
$10^{-5}$ & $1 \times 10^{6}$ & $3 \times 10^{-1}$ & $7 \times 10^{-8}$ \\
$10^{-7}$ & $1 \times 10^{4}$ & $3 \times 10^{-5}$ & $7 \times 10^{-14}$ \\
\hline \hline
\end{tabular}

readout noise of $0.14 e^{-} \mathrm{rms} /$ pixel (read time of 1.5 hours), which are read continuously for a total accumulated exposure time of one year. A threshold of $3 e^{-}$can be achieved for a dark-current value comparable to the upper limit reported in similar CCD detectors [15], while a dark current of $\lesssim 10^{-5} e^{-}$pixel $^{-1} /$ day $^{-1}$ would reduce the expected number of pixels with $\geq 2 e^{-}$from dark current to a negligible level. A selection cut is required to avoid misclassifying events in the right tail of the lowest charge bin dominated by dark-current events. For a noise of $0.14 e^{-} \mathrm{rms} /$ pixel, dark-current events can be rejected with a power of $\mathcal{O}\left(10^{9}\right)$ by requiring a charge measurement of $>0.84 e^{-}(>6 \sigma)$ above the dark-current bin (i.e., $1 e^{-}$or $2 e^{-}$), while maintaining a selection efficiency of $87 \%$ on the first signal bin ( $2 e^{-}$or $3 e^{-}$, respectively).

Applications.-Particle physics: The ability to precisely count the number of electrons in a pixel with a threshold as low as $2 e^{-}$or $3 e^{-}$has significant implications for rare event searches. In particular, the Skipper CCD allows for the construction of a new dark matter direct-detection experiment with unprecedented sensitivity to several classes of particle dark matter. This includes dark-matter particles with masses $\gtrsim 1 \mathrm{MeV}$ that scatter off an electron [21-24], and bosonic dark matter particles (pseudoscalar, scalar, or vector particles) with masses greater than a few $\mathrm{eV}$ that get absorbed by an electron [25-28]. Dark matter that scatters elastically off nuclei or that scatters inelastically off nuclei while emitting a photon [29], can produce a measurable signal in a Skipper CCD for dark matter masses as low as a few hundred $\mathrm{MeV}$ or a few tens of $\mathrm{MeV}$, respectively.

We detail one example. Sub-GeV dark matter can scatter off an electron in the valence band of silicon, promoting it to the conduction band. The typical recoil energy of the scattered electron is a few $\mathrm{eV}$, with the dark matter electron scattering rate falling steeply for larger recoil energies. Assuming that $3.6 \mathrm{eV}$ of energy above the silicon band gap is needed to create each additional electron-hole pair [30,31], a threshold of $2 e^{-}\left(3 e^{-}\right)$corresponds to an electron recoil energy of about 4.7 (8.3) eV. Such low thresholds would capture the majority of dark-matter electron-scattering events for dark-matter masses above
$\mathcal{O}(\mathrm{MeV})$ [21]. The readout noise of previous silicon CCD detectors required a threshold of $\sim 11 e^{-}(40 \mathrm{eV})$ [32], resulting in a relatively high mass threshold and limited cross-section sensitivity [21]. A $2 e^{-}$or $3 e^{-}$threshold in silicon is also lower than previously achieved using xenon time projection chambers (TPCs), whose threshold is set by the minimum ionization energy of xenon $(12.1 \mathrm{eV})$. Existing XENON10 data [33] constrain dark matter as light as $\sim 5 \mathrm{MeV}$ [34,35]; however, a detector-specific background limits the sensitivity and discovery potential of this experiment (it is unclear whether further research could reduce this background in xenon TPCs [36]). In contrast, a Skipper CCD experiment has significant discovery reach and is potentially background free for exposures up to $\sim 100$ gram years, assuming radiogenic background levels of $<1.5$ counts $/ \mathrm{kg} / \mathrm{keV} /$ day as achieved in other experiments [37-40]. Such a low rate will produce $<0.2$ background events in each charge bin of width $3.6 \mathrm{eV}$. Taking two or three charge bins, the experiment will capture most of the dark-matter signal and is expected to be background free.

In addition to dark-matter searches, CCD sensors have exciting applications as targets for coherent neutrino-nucleus scattering [41-43]. These experiments have a low event rate and do not require fast readout times. The lower energy threshold of Skipper CCDs would enable the exploration of new neutrino parameter space and nonstandard neutrino interactions [44].

Astronomy: Silicon CCDs have ushered in an era of precision astronomy and cosmology. To assess the impact of Skipper CCDs for astronomical applications, we examine how the SNR per pixel depends on the readout noise. Specifically, we define

$$
\mathrm{SNR}=\frac{r_{\mathrm{sig}} t_{\mathrm{exp}}}{\sigma_{\mathrm{tot}}}
$$

where $r_{\text {sig }}$ is the count rate of the signal source, $t_{\exp }$ is the exposure time, and $\sigma_{\text {tot }}$ is the total noise contribution per pixel,

$$
\begin{aligned}
\sigma_{\mathrm{tot}}^{2} & =\left(r_{\mathrm{sig}}+r_{\mathrm{bkg}}\right) t_{\mathrm{exp}}+r_{\mathrm{dark}} t_{\mathrm{exp}}+\sigma_{\text {read }}^{2}, \\
& =r t_{\text {exp }}+\sigma_{\text {read }}^{2} .
\end{aligned}
$$

Here, we have combined the Poisson shot noise from the signal source and sky background (including other sources of incident light) with the contribution from the dark current, $r=\left(r_{\text {sig }}+r_{\text {bkg }}+r_{\text {dark }}\right)$. The readout noise per pixel, $\sigma_{\text {read }}$, is independent of exposure time. To reach a given SNR threshold requires an exposure time expressed as,

$$
t_{\exp }=\frac{\mathrm{SNR}^{2} r}{2 r_{\mathrm{sig}}^{2}}\left(1+\sqrt{1+\frac{4 r_{\mathrm{sig}}^{2} \sigma_{\mathrm{read}}^{2}}{r^{2} \mathrm{SNR}^{2}}}\right) .
$$

The nondestructive readout of the Skipper CCD makes it possible to reduce the readout noise from $\sigma_{\text {read }}=\sigma_{1}$ to 
$\sigma_{\text {read }}=\sigma \ll 1$ (Eq. (1). A drastic reduction in readout noise can significantly reduce the exposure time required to reach a given SNR if

$$
\sigma_{1}>\frac{\mathrm{SNR} r}{2 r_{\text {sig }}} .
$$

Assuming a single-sample readout noise of $\sigma_{1}=$ $3.55 e^{-} \mathrm{rms} /$ pixel, the applications that will benefit most from reduced readout noise will be signal dominated, $r \approx r_{\text {sig }}$, but possess SNR $\lesssim 7$.

One exciting application in the low-SNR regime is direct, space-based imaging and spectroscopy of terrestrial exoplanets in the habitable zones of nearby stars [45]. The photon flux from exo-Earths is expected to be $\mathcal{O}(1)$ per several minutes, necessitating ultralow noise detectors [46]. A detector with subelectron readout noise could reduce exposure times by a factor of 2 , which is essential given the large exposure times required [47]. Skipper CCDs are easily manufactured with large formats and are stable over a large dynamic range. In addition, thick fully depleted CCDs can achieve high quantum efficiency between 0.87 and $1 \mu \mathrm{m}$ where several important spectral lines from water reside [46].

The authors gratefully acknowledge Juan Estrada for many useful conversations, and Chris Bebek for his contributions to the development of the Skipper CCD. The authors thank Ting $\mathrm{Li}$ for helpful discussions on astronomical applications. R.E. is supported by the U.S. DOE Early Career Research Program No. DESC0008061 and through a Sloan Foundation Research Fellowship. T. V. is supported by the I-CORE Program of the Planning Budgeting Committee and the Israel Science Foundation (Grant No. 1937/12), the European Research Council (ERC) under the EU Horizon 2020 Programme (ERCCoG-2015-Proposal No. 682676 LDMThExp), and the German-Israeli Foundation (Grant No. I-1283-303.7/ 2014). LBNL CCD development was supported by the Director, Office of Science, of the U.S. Department of Energy under Contract No. DE-AC02-05CH11231. This work was supported by Fermilab LDRD under U.S. DOE Contract No. DE-AC02-07CH11359.

*javiert@fnal.gov

Deceased.

[1] W. S. Boyle and G. E. Smith, Bell Syst. Tech. J. 49, 587 (1970).

[2] G. F. Amelio, M. F. Tompsett, and G. E. Smith, Bell Syst. Tech. J. 49, 593 (1970).

[3] C. Damerell, F. Farley, A. Gillman, and F. Wickens, Nucl. Instrum. Methods Phys. Res. 185, 33 (1981).

[4] J. R. Janesick, Scientific Charge Coupled Devices (SPIE Press, Bellingham, WA, 2001).

[5] M. H. White, D. R. Lampe, F. C. Blaha, and I. A. Mack, IEEE J. Solid-State Circuits 9, 1 (1974).

[6] J. Janesick and J. Tower, Sensors 16, 688 (2016).
[7] C. Bebek, J. Emes, D. Groom, S. Haque, S. Holland, P. Jelinsky, A. Karcher, W. Kolbe, J. Lee, N. Palaio, D. Schlegel, G. Wang, R. Groulx, R. Frost, J. Estrada, and M. Bonati, J. Instrum. 12, C04018 (2017).

[8] J. R. Janesick, T. Elliott, A. Dingizian, R. A. Bredthauer, and C. E. Chandler, Proc. SPIE Int. Soc. Opt. Eng. 1242, 223 (1990).

[9] D. Wen, IEEE J. Solid-State Circuits 9, 410 (1974).

[10] C. E. Chandler, R. A. Bredthauer, J. R. Janesick, J. A. Westphal, and J. E. Gunn, Proc. SPIE Int. Soc. Opt. Eng. 1242, 238 (1990).

[11] G. Fernandez Moroni, J. Estrada, G. Cancelo, S. E. Holland, E. E. Paolini, and H. T. Diehl, Exp. Astron. 34, 43 (2012).

[12] G. Lutz, M. Porro, S. Aschauer, S. Wölfel, and L. Strüder, Sensors 16, 608 (2016).

[13] S. Holland, Nucl. Instrum. Methods Phys. Res., Sect. A 275, 537 (1989).

[14] S. E. Holland, D. E. Groom, N. P. Palaio, R. J. Stover, and M. Wei, IEEE Trans. Electron Devices 50, 225 (2003).

[15] A. Aguilar-Arevalo et al. (DAMIC Collaboration), Phys. Rev. Lett. 118, 141803 (2017).

[16] B. L. Flaugher et al., Proc.SPIE 8446, 844611 (2012).

[17] B. Flaugher et al. (DES Collaboration), Astron. J. 150, 150 (2015).

[18] M. S. Haro, G. Cancelo, G. F. Moroni, X. Bertou, J. Tiffenberg, E. Paolini, and J. Estrada, in Proceedings of the 2016 Argentine Conference of Micro-Nanoelectronics, Technology and Applications (CAMTA), Argentina, 2016 (IEEE, Piscataway, NJ, 2016), pp. 11-16.

[19] P. O'Connor, J. Instrum. 10, C05010 (2015).

[20] D. Doering, N. Andresen, D. Contarato, P. Denes, J. M. Joseph, P. McVittie, J. P. Walder, J. Weizeorick, and B. Zheng, in Proceedings of the 2011 IEEE Nuclear Science Symposium Conference Record, Valencia, Spain, 2011 (IEEE, Piscataway, NJ, 2011), pp. 1840-1845.

[21] R. Essig, M. Fernandez-Serra, J. Mardon, A. Soto, T. Volansky, and T.-T. Yu, J. High Energy Phys. 05 (2016) 046.

[22] R. Essig, J. Mardon, and T. Volansky, Phys. Rev. D 85, 076007 (2012).

[23] P. W. Graham, D. E. Kaplan, S. Rajendran, and M. T. Walters, Phys. Dark Universe 1, 32 (2012).

[24] S. K. Lee, M. Lisanti, S. Mishra-Sharma, and B. R. Safdi, Phys. Rev. D 92, 083517 (2015).

[25] H. An, M. Pospelov, and J. Pradler, Phys. Rev. Lett. 111, 041302 (2013).

[26] H. An, M. Pospelov, J. Pradler, and A. Ritz, Phys. Lett. B 747, 331 (2015).

[27] I. M. Bloch, R. Essig, K. Tobioka, T. Volansky, and T.-T. Yu, J. High Energy Phys. 06 (2017) 087.

[28] Y. Hochberg, T. Lin, and K. M. Zurek, Phys. Rev. D 95, 023013 (2017).

[29] C. Kouvaris and J. Pradler, Phys. Rev. Lett. 118, 031803 (2017).

[30] C. A. Klein, J. Appl. Phys. 39, 2029 (1968).

[31] B. Streetman and S. Banerjee, Solid State Electronic Devices, (Pearson, Boston, 2005), 7th ed.

[32] J. Barreto et al. (DAMIC Collaboration), Phys. Lett. B 711, 264 (2012).

[33] J. Angle et al. (XENON10 Collaboration), Phys. Rev. Lett. 107, 051301 (2011); 110, 249901(E) (2013). 
[34] R. Essig, A. Manalaysay, J. Mardon, P. Sorensen, and T. Volansky, Phys. Rev. Lett. 109, 021301 (2012).

[35] R. Essig, T. Volansky, and T.-T. Yu, Phys. Rev. D 96, 043017 (2017).

[36] P. Sorensen, arXiv:1702.04805.

[37] R. Agnese et al. (SuperCDMS Collaboration), Phys. Rev. Lett. 116, 071301 (2016).

[38] C. E. Aalseth et al. (CoGeNT Collaboration), Phys. Rev. D 88, 012002 (2013).

[39] N. Abgrall et al. (Majorana Collaboration), Phys. Rev. Lett. 118, 161801 (2017).

[40] E. Armengaud et al. (EDELWEISS Collaboration), Astropart. Phys. 91, 51 (2017).

[41] G. Fernandez Moroni, J. Estrada, E. E. Paolini, G. Cancelo, J. Tiffenberg, and J. Molina, Phys. Rev. D 91, 072001 (2015).
[42] A. Aguilar-Arevalo et al., J. Phys. Conf. Ser. 761, 012057 (2016).

[43] A. Aguilar-Arevalo et al. (CONNIE Collaboration), J. Instrum. 11, P07024 (2016).

[44] R. Harnik, J. Kopp, and P. A. N. Machado, J. Cosmol. Astropart. Phys. 07 (2012) 026.

[45] National Research Council, New Worlds, New Horizons in Astronomy and Astrophysics (The National Academies Press, 2010).

[46] B. Crill and N. Siegler (2017), JPL Document D-98883.

[47] S. Shaklan, M. Levine, M. Foote, M. Rodgers, M. Underhill, L. Marchen, and D. Klein, in Techniques and Instrumentation for Detection of Exoplanets VI, Proc. SPIE, Vol. 8864 (2013) p. 886415. 